\title{
Italian national survey on molecular epidemiology of Pseudomonas aeruginosa from pulmonary infection of Cystic Fibrosis patients
}

\author{
Graziana Manno', Patrizia Morelli', Flavio Favari², Maria Laura Garlaschi', Lisa Cariani ${ }^{3}$, \\ Ersilia Fiscarelli', Esther Manso ${ }^{5}$, Natalia Cirilli ${ }^{5}$, Angelica D’Aprile ${ }^{6}$, Fulvia Gioffrè \\ Donatella Scuderi' ${ }^{7}$ Ivana Collebrusco ${ }^{8}$,Tatiana Borio ${ }^{8}$ \\ I Laboratorio Generale Analisi e Microbiologia - Sezione Microbiologia della Fibrosi Cistica, Istituto Giannina Gaslini e \\ Dipartimento di Scienze Pediatriche, Università di Genova, \\ 2 Ospedale Borgo Trento - Verona, \\ 3 Istituti Clinici di Perfezionamento - Milano, \\ 4 Ospedale Bambin Gesù Roma, \\ 5 Ospedale di Ancona, \\ 6 Ospedale di Cerignola, \\ 7 Ospedale di Soverato, \\ 8 Ospedale di Gualdo Tadino
}

Key words: P. aeruginosa, Cystic Fibrosis, Genetic fingerprinting

Epidemiolgia molecolare italiana di Pseudomonas aeruginosa in Fibrosi Cistica

\section{SUMMARY}

Pulmonary infection sustained by Pseudomonas aeruginosa is the leading cause of morbidity and mortality of patients affected by Cystic Fibrosis (FC), the most common inherited disease among Caucasian. P. aeruginosa is a ubiquitous microorganism, widely diffuse in the natural and nosocomial environment; however the initial source of acquisition and the way of transmission of this pathogen among CF patients are not yet elucidated. The acquisition of $P$. aeruginosa by the environment and/or patients to patients transmission was speculated. Moreover in some countries (Australia, United Kingdom) some highly virulent and transmissible strains, called epidemic strains, have spread within the CF care Centres. Aim of the present study was to determine the presence of possible diffuse lineages and the cross-infection degree of $P$. aeruginosa among Italian $C F$ patients. For this purpose we carried out a national survey, determining the genetic relationship, by molecular typing of a collection of 530 strains recovered from respiratory specimens of 335 patients colonised in follow-up at 8 Regional Reference CF Care Centres located in 3 geographic Italian areas. To investigate a possible environmental acquisition of $P$. aeruginosa, the genetic relationship between strains from FC, from natural environment, household and nosocomial sources. The molecular epidemiological survey was assessed using DNAfingerprinting methods (Box-PCR e Multi Locus Sequence Typing).

\section{INTRODUZIONE}

La fibrosi cistica (FC) o mucoviscidosi è la malattia congenita, cronica, evolutiva, trasmessa con meccanismo autosomico recessivo, più frequente nella popolazione caucasica. In Italia la frequenza stimata è di 1 malato ogni 2500 nati vivi. Attualmente in Italia sono presenti 3500 individui affetti seguiti presso 40 centri di Cura regionali dedicati. Il gene responsabile della FC è localizzato in una regione del braccio lungo del cromosoma 7; questo gene codifica per una complessa proteina di membrana denominata CFTR (Cystic Fibrosis Transmembrane Conductance Regulator) la cui funzione è di regolare il flusso degli ioni $\mathrm{Cl}^{-}$ attraverso le membrane apicali delle cellule epite- liali con conseguente disidratazione delle secrezioni mucose polmonari e difetto delle modalità fisiologiche di "clearence"; questa condizione favorisce l'adesione di microrganismi, uno stato infiammatorio cronico conseguente ad una colonizzazione persistente (22). Gli agenti etiologici dell'infezione polmonare in FC sono Staphylococcus aureus, Haemophilus influenzae, Burkholderia cepacia -complex, miceti, ma è Pseudomonas aeruginosa il patogeno riconosciuto come la causa più rilevante del danno polmonare progressivo (24). Durante la colonizzazione cronica, $P$. aeruginosa va incontro a fenomeni di adattamento in cui attua dei cambiamenti fenotipici: si ha la comparsa nelle colture microbiologiche

\section{Corresponding author: Graziana Manno}

Dip.to di Scienze Pediatriche, Università di Genova, Lab. Generale di Analisi e Microbiologia, Istituto G. Gaslini, Largo G. Gaslini 5 - 16147 Genova - Tel.: +300105636314

Email: graziana.manno@unige.it 
delle secrezioni respiratorie di questi pazienti di ceppi mucoidi per produzione di alginato (19), di ceppi auxotrofi e Small Colony Variant (SCV) (29); inoltre il microrganismo è portato ad esprimere elevati gradi di resistenza agli antibiotici. I pazienti affetti da FC nel corso della loro vita ed a causa del tipo di malattia cronica, sono soggetti a frequenti ospedalizzazioni che rappresentano un rischio di esposizione a fonti esogene di $P$. aeruginosa, come altri pazienti FC colonizzati dal microrganismo e/o l'ambiente nosocomiale (7).

Tuttavia la fonte iniziale di $P$. aeruginosa e le modalità di trasmissione tra pazienti affetti da FC non sono ancora stabiliti con chiarezza. P. aeruginosa è un germe ubiquitario che predilige gli ambienti umidi, può essere ritrovato nel suolo, nell'acqua e nelle piante. È in grado di sopravvivere nell'acqua distillata e in svariate soluzioni acquose quali disinfettanti, detergenti, soluzioni per irrigazione, soluzioni ed equipaggiamenti per dialisi, ed è inoltre possibile isolarlo da ossigenatori, sifoni dei lavandini e nebulizzatori per aerosol terapia e dalle docce (18). In aggiunta alle fonti nosocomiali, $P$. aeruginosa può essere isolato anche da piscine, tubature dei riscaldamenti, soluzioni per lenti a contatto, unghie finte, cosmetici e suole interne delle scarpe da ginnastica (20). La presenza ubiquitaria di $P$. aeruginosa in natura e in ambiente ospedaliero può spiegare l'elevata frequenza con cui i pazienti FC acquisiscono questo microrganismo. Quindi gli studi volti alla comprensione delle dinamiche attraverso cui pazienti $\mathrm{FC}$ acquisiscono $P$. aeruginosa sono di fondamentale importanza, sia per il rilevante impatto che l'infezione polmonare sostenuta riveste nella storia naturale della malattia, sia per il miglioramento delle misure di prevenzione e controllo dell'infezione. Attualmente le moderne tecniche di tipizzazione intraspecie, che utilizzano markers genetici (DNA-fingerprinting), hanno fornito uno strumento importante per indagare le possibili fonti d'infezione, le modalità di acquisizione, la possibile trasmissione interpersonale e anche l'evoluzione del patogeno nel paziente colonizzato (28). Vi sono evidenze che la prima acquisizione possa avvenire dall'ambiente, infatti, un complesso clonale di $P$. aeruginosa altamente diffuso, denominato Clone $\mathrm{C}$, è stato ritrovato sia nell'ambiente naturale sia in un ampio spettro di tipologie di infezioni, compresa quella polmonare in FC (5). Al contrario, altri studi suggeriscono che la fonte di acquisizione iniziale sia rappresentata da alcuni ceppi clonali diffusi tra la popolazione FC, attraverso infezioni crociate (14). A questi studi si sono affiancate altre ricerche volte a chiarire quale sia il reale grado di trasmissibilità di questo germe, tuttavia non si è arrivati a una conclusione univoca, dal momento che i risultati ottenuti non sono concordi. A tal proposito due grandi studi di epidemiologia molecolare condotti in Centri FC in Australia (21) e in Canada (27) hanno portato risultati diametralmente opposti: nello specifico il gruppo australiano riporta una vasta diffusione di ceppi clonali all'interno di un centro FC pediatrico, al contrario il gruppo canadese non ha evidenziato una così frequente trasmissione di ceppi fra pazienti, se non dopo un prolungato e stretto contatto tra loro. In diversi paesi Europei, sono stati inoltre riportati altri casi di cross-infezioni di P. aeruginosa in centri di riabilitazione e campi estivi dedicati ai pazienti FC. Altri studi, Liverpool e Manchester (26) ancora riportano una diffusione di ceppi con genotipo identico all'interno dei centri FC, definiti ceppi epidemici o altamente trasmissibili. I pazienti infettati con ceppi epidemici hanno mostrato un decorso clinico peggiore rispetto a quelli infetti con ceppi non epidemici, compreso un maggior numero di ospedalizzazioni e ripetuti cicli di trattamenti antibiotici (17). Gli studi epidemiologici, che si propongono di approcciare in modo efficace la problematica di $P$. aeruginosa in FC, dovrebbero avere come supporto di indagine la tipizzazione genetica condotta mediante metodiche affidabili di biologia molecolare. La tipizzazione molecolare comprende un insieme di metodiche finalizzate allo studio dei genomi batterici, al fine di rilevarne il grado di diversità/similarità molecolare per evidenziare la presenza o diffusione di cloni all'interno di un dato ambiente. Le tecniche di tipizzazione sono inoltre importanti per evidenziare l'emergenza di nuovi organismi ipervirulenti o multifarmaco-resistenti. L'epidemiologia molecolare dei microrganismi si è sviluppata in conseguenza della definizione del concetto di clonalità. Il termine Clone viene adottato per indicare colture batteriche isolate indipendentemente, da differenti fonti, da differenti aree, e anche in tempi differenti, ma che dimostrano talmente tanti tratti genotipici identici da giustificare questa identità attribuendo un'origine comune. Attualmente metodi di tipizzazione che si basano sullo studio di caratteri genetici, sono riconosciuti come il mezzo di riferimento per determinare l'epidemiologia molecolare di microrganismi diffusi nell'ambiente naturale e nosocomiale, in infezioni comunitarie e ospedaliere compresa la FC. Questi metodi sono caratterizzati da differenti poteri di discriminazione, target genetici e analisi dei profili e l'interpretazione dei risultati. Ad oggi numerosi metodi molecolari (PFGE, RAPD, RFLP, ecc) sono stati proposti nella tipizzazione di $P$. aeruginosa (12). Tra tutte le metodiche elencate, la BoxPCR rappresenta una tecnica particolarmente ido- 
nea, per le caratteristiche di semplicità, rapidità, riproducibilità, potere discriminatorio ed economicità per il typing molecolare di $P$. aeruginosa. Tale metodica si basa sull'amplificazione di sequenze chiamate sequenze REP (Ripetute, Extrageniche e Palindrome) che in $P$. aeruginosa sono definite elementi Box. La metodica di BoxPCR permette quindi di ottenere una serie di bande con peso molecolare variabile che, visualizzate su gel elettroforesi, creano un profilo univoco che ricorda un codice a barre (barcode). Tali bande possono essere confrontate mediante l'utilizzo di software dedicati che permettono di analizzare il grado di similarità dei diversi profili molecolari inseriti. Recentemente è stata sviluppata una promettente tecnica di tipizzazione molecolare denominata Multi Locus Sequence Typing (MLST); tale tecnica è in grado di caratterizzare in modo non ambiguo ceppi microbici tramite il sequenziamento nucleotidico di frammenti interni di geni denominati housekeeping, specie-specifici, solitamente sette, che sono altamente conservati e codificano per importanti attività metaboliche. La MLST consiste in una prima amplificazione ed un successivo sequenziamento dei geni housekeeping. Le sequenze ottenute sono poi inserite in un database dedicato (http://pubmlst.org/paeruginosa/) in cui un apposito software (Bionumerics) assegna un codice numerico definito Sequenza Tipo (ST). Queste sequenze sono portabili e quindi confrontabili con altre ST immesse nel database. La MLST è una tecnica adatta a studi di evoluzione e diffusione delle popolazioni batteriche e permette di confrontare l'epidemiologia di un singolo patogeno a livello sovra-locale, ma non è idonea a studi su epidemie localizzate. Nel presente studio multicentrico è stata indagata l'epidemiologia molecolare italiana di $P$. aeruginosa in FC mediante la determinazione della correlazione genetica di isolati di P. aeruginosa da pazienti in follow up presso otto centri di cura dislocati in diverse aree geografiche (Nord, Centro e Sud), e dell'eventuale presenza di ceppi epidemici e/o altamente trasmissibili. Inoltre, per delucidare una possibile acquisizione ambientale, è stata anche determinata la correlazione genetica dei ceppi di $P$. aeruginosa FC con ceppi di origine ambientale naturale, domestico e nosocomiale.

\section{MATERIALI E METODI \\ Ceppi batterici, Centri FC e pazienti}

Presso il Laboratorio Generale di Analisi e Microbiologia, sezione Microbiologia della Fibrosi Cistica, dell'Istituto Giannina Gaslini di Genova, durante il 2005-2007 sono stati collezionati un totale di 530 ceppi di $P$. aeruginosa isola- ti da 335 pazienti colonizzati; per ogni paziente sono stati selezionati i diversi morfotipi presenti (mucoide e/o non mucoide e/o SCV).

I centri FC partecipanti allo studio sono Genova (281 ceppi/117 pazienti), Ancona (40 ceppi/36 pazienti), Cerignola (40 ceppi/ 36 pazienti), Soverato (20 ceppi/ 17 pazienti), Gualdo Tadino (23 ceppi/ 18 pazienti), Verona (30 ceppi/ 30 pazienti), Milano (72 ceppi/ 38 pazienti), Roma Ospedale Bambin Gesù (66 ceppi / 43 pazienti). I ceppi sono stati isolati ed identificati con le metodiche per i campioni FC (11), e successivamente conservati a $-80^{\circ} \mathrm{C}$ in Vials (Nalgene, Germania) contenenti $800 \mathrm{mcl}$ di Tryptic Soy Broth (Difco, USA) e $200 \mathrm{mcl}$ di glicerolo.

\section{Ceppi di riferimento}

E stato inserito un panello di ceppi di riferimento/epidemici europei: PA ATCC 27853, PA01 (30), Clone C (25), Liverpool Epidemic Strain (LES) Manchester Epidemic Strain (MES) (31).

\section{Ceppi ambientali}

Sono stati anche collezionati e analizzati 55 ceppi da campioni ambientali, di cui 30 ceppi da 7 piscine della provincia di Genova, 25 ceppi da lavandini domestici e nosocomiali.

\section{Genotipizzazione}

a) Box-PCR. Si è proceduto come precedentemente descritto (23), brevemente (Figura I): per l'estrazione del DNA batterico, da una coltura overnight sono state prelevate 1-2 colonie e inoculate in $20 \mu \mathrm{ldi}$ un tampone di lisi contenente $0.25 \%$ (peso/vol) di sodio dodecil solfato (SDS) e $0.05 \mathrm{~N}$ $\mathrm{NaOH}$. Dopo una bollitura a $95^{\circ} \mathrm{C}$ per 10 minuti, sono stati aggiunti $180 \mu \mathrm{l}$ di acqua distillata sterile. Per l'amplificazione sono state effettuate alcune modifiche al protocollo proposto. Il primer utilizzato è stato BOX-A1R (5'-CTACGGCAAGGCGACGCTGACG-3'). La reazione di PCR è stata effettuata in $25 \mu \mathrm{l}$ finali. Cicli di amplificazione: iniziale denaturazione a $95^{\circ} \mathrm{C}$ per 2' sono stati eseguiti 35 cicli composti da $94^{\circ} \mathrm{C}$ per 3 ", $92^{\circ} \mathrm{C}$ per $30^{\prime \prime}, 50^{\circ} \mathrm{C}$ per 1 ', estensione finale $65^{\circ} \mathrm{C}$ per $8^{\prime}$. I prodotti di PCR sono poi stati separati in un gel con corsa elettroforetica di $18 \mathrm{~h}$ a 45 Volt. Quindi i gel sono stati visualizzati mediante raggi UV e le immagini ottenute sono state anche digitalizzate.

b) Analisi e comparazione con software Gel Compare II. Ciascuna immagine del profilo molecolare è stata convertita e quindi normalizzata. Per definire il grado di similarità tra ciascun profilo è stato usato il coefficiente di Pearson e la cluster analisi della matrice di similarità è stata generata usando il metodo UPGMA (Unweighted Pair Group Method with Arithmetic Mean). Come limite di similarità per definire $i$ cloni tra i profili esaminati è stato scelto almeno il $97 \%$, di bande 
simili. I cluster sono stati definiti come gruppi di pazienti o campioni ambientali che condividono lo stesso genotipo di P. aeruginosa.

$M L S T$. L'analisi è stata effettuata come precedentemente descritto (4) e come suggerito nel sito dedicato http://pubmlst.org/paeruginosa/. Lo schema per "P. aeruginosa MLST" usa dei frammenti interni dei seguenti sette geni "house-keeping": acsA (Acetyl coenzima A sintetasi), aroE (Shikimate dehydrogenasi), guaA (GMP sintasi), mutL (DNA mismatch repair protein), nuoD (NADH dehydrogenase I chain C, D), ppsA (Phosphoenolpyruvate synthase), trpE (Anthralite synthetase component I). I profili di sequenza sono stati ottenuti con il sequenziatore automatico ABI3100 (Applied-Biosystem) e in seguito comparati con i profili presenti nel sito web MLST $P$. aeruginosa per mezzo del programma di bioinformatica disponibile online.

\section{Indice statistico di bio-diversità}

Per definire il grado di Biodiversità tra i genotipi di $P$. aeruginosa $\mathrm{FC}$ e ambientali, si è utilizzato l'indice di Simpson (13), i cui valori ricadono in un range compreso tra 0 e 1 , dove 0 indica assenza di variabilità, mentre 1 rappresenta il massimo grado di variabilità.

\section{RISULTATI}

Box-PCR. La Figura II è rappresentativa della cluster-analisi dei profili molecolari su gel ottenuti con la metodica e i parametri di analisi utilizzati.

\section{Epidemiologia molecolare ceppi FC}

La Figura III riassume i risultati trovati in totale su tutti i centri analizzati. Il primo diagramma (Pazientin. Genotipi) indica che $1 ' 80 \%$ dei pazienti, pur avendo colture positive per diversi morfotipi, è colonizzato da un solo genotipo di $P$. aeruginosa. I diagrammi riportano il grado di correlazione fra i diversi genotipi: il $55 \%$ dei pazienti alberga un genotipo unico e distinto, mentre il $45 \%$ dei pazienti è colonizzato da un genotipo che è correlato, condiviso con altri pazienti con cui forma dei gruppi (cluster); il 70\% dei cluster è formano da solo 2 pazienti.

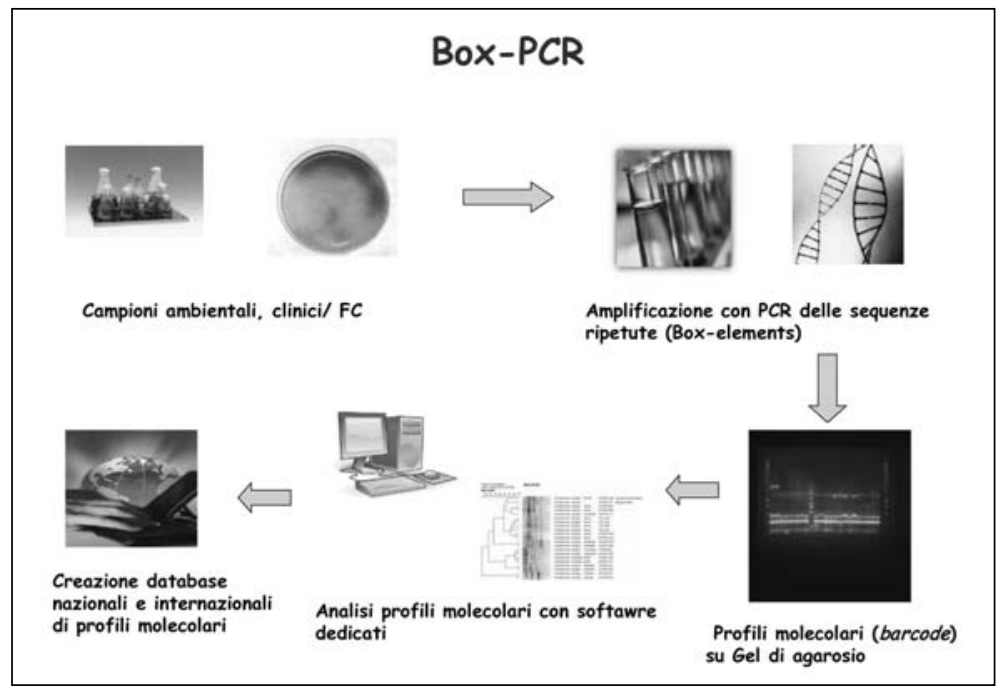

Figura I. Schema del protocollo della metodica di typing molecolare BoxPCR. La metodica Box- PCR si basa sull'amplificazione, con PCR mediante l'uso di un primer arbitrario, di sequenze ripetute (elementi Box per P. aeruginosa) cosi da ottenere una serie di bande con peso molecolare variabile che, visualizzate su gel elettroforesi, creano un profilo univoco che ricorda un codice a barre (barcode). Tali sequenze di bande possono essere confrontate e messe in relazione mediante l'utilizzo di software dedicati che permettono di analizzare il grado di similarità dei diversi profili molecolari inseriti. Inoltre è possibile creare delle banche dati dei genotipi ottenuti.

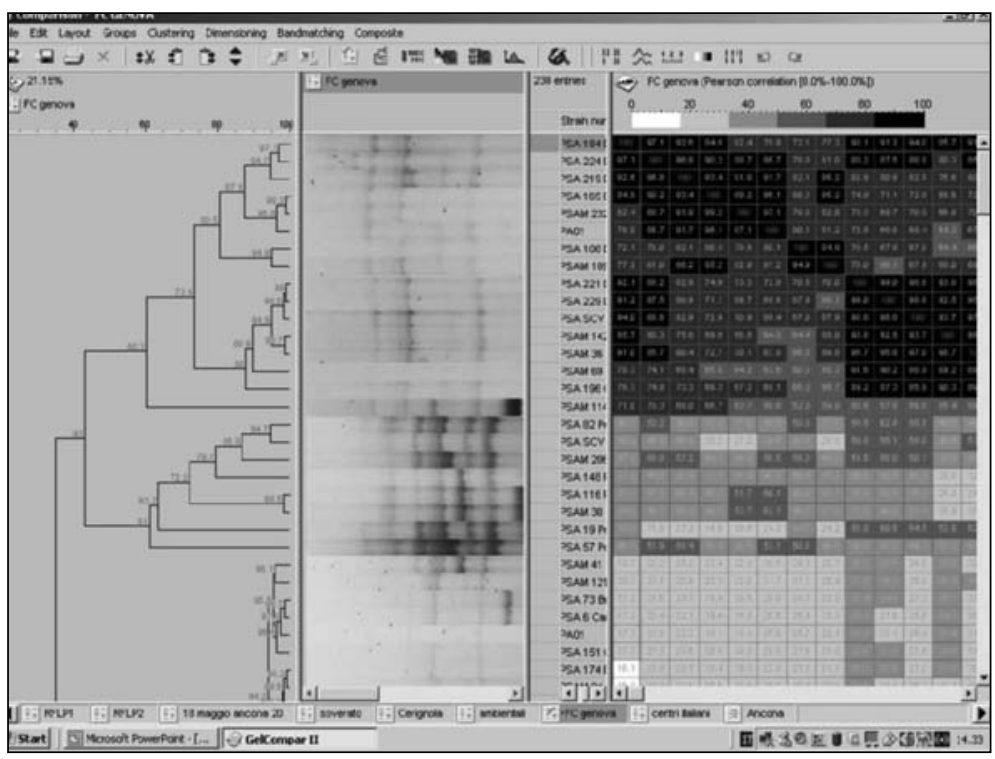

Figura II. Analisi dei profili molecolari utilizzando Gel Compare II.

Questo programma di analisi permette di analizzare le dei profili molecolari visualizzati su gel di agarosio e di compararli fra loro, calcolando mediante il Coefficiente di Pearson il grado di similarità dei genotipi. Inoltre si possono creare dei dendrogrammi con cluster analisi utilizzando il sistema aritmetico (UPGMA). Si possono osservare i profili di ceppi FC confrontati.

\section{Epidemiologia molecolare ceppi ambientali}

Sono stati individuati 25 genotipi/ 55 ceppi analizzati: 12 genotipi sono unici e non correlano con nessun altro genotipo dei ceppi ambientali analizzati. Diversamente 13 genotipi correlano fra loro e formano i seguenti cluster: 10 cluster da 2 sorgenti ambientali; 3 cluster da 3 sorgenti ambientali.

Comparazione tra i profili molecolari fra i 
ceppi FC i ceppi ambientali e i ceppi epidemici Europei

Tutti i profili molecolari, ottenuti mediante Box-PCR, dei diversi centri FC studiati, sono stati comparati fra loro. Questa analisi ha messo in evidenza una correlazione genetica solo nel caso di 3 pazienti del centro di Ancona con 2 pazienti del centro di Soverato. Questo gruppo di pazienti è stato definito Gruppo Clonale Italiano FC (GCIFC) ed è stato ulteriormente tipizzato mediante MLST (vedi sotto). Non si è trovata nessuna correlazione genetica tra i profili molecolari appartenenti ai ceppi isolati dai pazienti FC e i ceppi isolati dall'ambiente.

Per quanto concerne la comparazione dei profili genetici dei ceppi FC e del GCI-FC e dei ceppi ambientali italiani con i maggiori ceppi Epidemici circolanti in Europa (LES, MES e Clone C) non è stato possibile evidenziare correlazioni genetiche con il coefficiente di similarità da noi usato del $97 \%$.

\section{MLST del GCI-FC}

Il sequenziamento mediante MLST del ceppo appartenente al gruppo clonale individuato in questo studio ha portato a definire la seguente Sequenza Tipo (ST):

\begin{tabular}{lccccccc} 
Geni & Acs & Aro & Gua & Mut & Nuo & Pps & Trp \\
\hline ST & 39 & 35 & 71 & 81 & 56 & 36 & 54
\end{tabular}
Tale ST è stata inserita nel database del sito MLST dedicato a PA, in cui sono presenti 385 ST di ceppi clonali ed epidemici tipizzati e caratterizzati in tutto il mondo. Questa ultima analisi ha escluso la correlazione del clone individuato con altri profili presenti nel database.

Biodiversità. Nella tabella (Tabella 1) sono riassunti i risultati sul grado di biodiversità, dei geno-

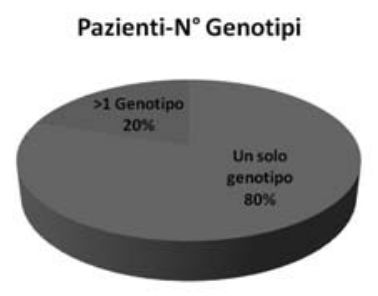

Figura III. Risultati sui genotipi di P. aeruginosa.

I diagrammi riportati riassumo i risultati ottenuti dall'analisi dei genotipi dei Pe dei Centri di Cura analizzati. II primo diagramma (Pazienti-n. morfotipi, è colonizzato da un solo genotipo di P. aeruginosa. Gli altri diagrammi ortano il grado di correlazione fra i diversi genotipi: il 55\% dei pazient un genotipo unico e distinto, mentre il $45 \%$ dei pazienti è colonizzato da un genotipo che è correlato, condiviso con altri pazienti con cui forma dei gruppi (cluster); il 70\% dei cluster è formano da solo 2 pazienti.

tipi ottenuti, calcolata mediante l'indice di Simpson. I valori dell'indice D ottenuti (range $=0.02-0.18$ ) indicano un'ampia variabilità genotipica nella popolazione analizzata.

\section{DISCUSSIONE E CONCLUSIONI}

Questo studio rappresenta la prima vasta indagine nazionale italiana sull'epidemiologia molecolare di $P$. aeruginosa isolati da pazienti con FC. I risultati si riferiscono, infatti, all'analisi di 530 isolati provenienti da 335 pazienti colonizzati da $P$. aeruginosa in follow up presso otto Centri Italiani per la Cura della FC dislocati in aree diverse (Nord, Centro e Sud Italia). È da sottolineare che sino ad oggi sono stati realizzati a livello italiano soli 4

Tabella I. Grado di Biodiversità dei genotipi di P. aeruginosa ottenuti dall'analisi dei ceppi FC e ambientali. La biodiversità tra i genotipi analizzati nello studio è stata calcolata con l'indice di Simpson: il valore dell'indice $D$ ha un range compreso tra 0 e $I$, dove 0 indica assenza di variabilità, mentre I rappresenta il massimo grado di variabilità. $I$ valori dell'indice $D$ ottenuti (range $=0.02-0.18$ ) indicano un'ampia variabilità genotipica nella popolazione analizzata.

\begin{tabular}{ccc}
\hline CENTRI FC & INDICE DI SIMPSON (D) & INDICE DI DIVERSITÁ DI SIMPSON (I-D) \\
\hline ANCONA & 0.13 & 0.87 \\
\hline CERIGNOLA & 0.18 & 0.82 \\
\hline SOVERATO & 0.16 & 0.84 \\
\hline GENOVA & 0.02 & 0.98 \\
\hline MILANO & 0.06 & 0.94 \\
\hline ROMA & 0.04 & 0.96 \\
\hline VERONA & 0.05 & 0.95 \\
\hline AMBIENTALI & 0.08 & 0.92 \\
\hline
\end{tabular}


studi di epidemiologia molecolare di $P$. aerugino$s a$ in FC; questi studi sono stati condotti analizzando un numero limitato di ceppi provenienti da pazienti seguiti in singoli centri $(1,2,8,15)$ utilizzando metodiche di fingerprinting diverse tra loro rendendo quindi difficile la comparazione dei dati e la reale comprensione dell'epidemiologia molecolare. Su questa tematica anche a livello europeo sono stati condotti un gran numero di studi, locali e nazionali, che si sono avvalsi di una varietà di metodiche di typing, caratterizzate da un diverso potere discriminatorio. Ad oggi la Pulsed Field Gel Eelettophoresis (PFGE) è ritenuta il gold standard, tuttavia questa metodica presenta alcune limitazioni in quanto è complessa, richiede strumentazioni altamente specifiche e costose, spazi, tempo e personale dedicato. Per le sue caratteristiche tecniche la PFGE non è stata mai applicata in studi di comparazione fra isolati di $P$. aeruginosa provenienti da aree geografiche differenti. Tra le metodiche di typing la Box-PCR è stata utilizzata in alcuni importanti studi su patogeni batterici della FC (3) e inoltre, in uno studio di comparazione con la PFGE, condotto in Australia su isolati di $P$. aeruginosa da pazienti $\mathrm{FC}$, sono stati ottenuti risultati sovrapponibili tra le due metodiche (31). La Box-PCR si è anche dimostrata efficace nel discriminare isolati di $P$. aeruginosa provenienti da diverse fonti (FC, cliniche non FC e/o ambientali) e aree geografiche (6). Tale tecnica, per le sue caratteristiche di rapidità, alto potere discriminatorio è riproducibilità è risultata idonea per lo studio di epidemiologia molecolare in FC di P. aeruginosa a livello nazionale.

Per superare la soggettiva dell'interpretazione del fitto bandeggio che si ottiene con questa metodica di typing, nel nostro studio ci si è avvalso di un software dedicato (Gel Compare) in grado di calcolare precisamente un coefficiente di correlazione (Pearson) indispensabile per la definizione della correlazione genetica di profili distinti. Tale dato, espresso in percentuale (da 0 a 100) indica la similarità fra due o più ceppi; risulta quindi importante la scelta di definire un limite al di sopra del quale due profili molecolari sono ritenuti identici. A tal fine si è scelto il valore di $97 \%$, come suggerito da precedenti studi che hanno valutato l'applicazione di tale parametro nell'analisi di Box-PCR.

La tipologia d'infezione respiratoria e la centralizzazione delle cure rivolte ai pazienti FC aumentano la possibilità di una reale diffusione di ceppi batterici all'interno delle strutture nosocomiali dedicate e come conseguenza una maggiore possibilità di cross-infezione tra $\mathrm{i}$ pazienti afferenti a tali strutture. La diffusione di ceppi batterici clo- nali in grado di cross-infettare e sovrainfettare $\mathrm{i}$ pazienti $\mathrm{FC}$ è già stata ampiamente dimostrata per un altro cruciale patogeno del polmone $\mathrm{FC}, B$. cepacia -complex (16). La politica di segregare $\mathrm{i}$ pazienti infettati da B. cepacia- complex ha dimostrato nel corso di questi anni di essere in grado di limitare la trasmissione di questo patogeno ad altri pazienti FC (9). Tuttavia il ruolo della segregazione nel ridurre la diffusione di P.aeruginosa all'interno dei centri FC rimane ad oggi ancora un argomento dibattuto. I risultati finora ottenuti dagli studi condotti a livello Europeo sulla trasmissibilità di $P$. aeruginosa fra pazienti FC non portano a conclusioni univoche. I risultati si possono dividere in due filoni contrapposti: alcuni autori che riportano un'importante diffusione di ceppi epidemici e la maggior parte degli studi che non segnalano una rilevante trasmissione di $P$. aeruginosa all'interno dei centri di cura. La presenza di ceppi epidemici, altamente trasmissibili o ceppi clonali, è stata ampiamente documentata in paesi quali il Regno Unito e l'Australia.

In linea quindi con la maggior parte degli studi nazionali di tipizzazione molecolare di P. aeruginosa condotti fino ad ora, nel nostro studio multicentrico il $45 \%$ dei pazienti condivide uno stesso genotipo con altri pazienti di uno stesso centro di cura e per la maggior parte dei casi $(70 \%$ dei cluster) vi è una condivisione tra due pazienti. Questo ci fa ipotizzare che anche nei centri analizzati siano avvenute delle cross-infezioni, dovute prevalentemente a limitati casi di stretti e/o prolungati contatti tra pazienti, dovute probabilmente ad improprie applicazioni delle norme previste per la prevenzione e il controllo delle infezioni dei patogeni respiratori in FC (10). Risultato rilevante del nostro studio è l'esclusione nei nostri Centri FC in Italia della diffusione di ceppi epidemici e della presenza dei maggiori cloni epidemici europei. Nel nostro studio è stata valutata inoltre la possibilità di un'eventuale acquisizione ambientale, sia naturale che nosocomiale, analizzando e confrontando fra loro e con quelli FC, i profili molecolari dei genotipi di ceppi di $P$. aeruginosa isolati da piscine, scarichi di lavandini domestici e nosocomiali. L'analisi genetica dei ceppi ambientali esclude che tra questi isolati vi siano ceppi clonali largamente diffusi e/o prevalenti. Inoltre i nostri risultati non hanno evidenziato correlazione genetica tra i diversi profili clinici ed ambientali. Questo dato porta ad escludere che i pazienti analizzati abbiano acquisito $P$. aeruginosa dall'ambiente, anche se è da sottolineare che il numero di ceppi ambientali raccolti ed esaminati è circoscritto all'area ligure, e quindi rapportabile solo ai pazienti in follow-up nel Centro FC di Genova e residenti in Liguria. Tali ceppi sono stati confron- 
tati anche con il profilo molecolare del clone epidemico ambientale (Clone $\mathrm{C}$ ) anche tale raffronto non ha messo in evidenza alcuna relazione genetica. Altro dato importante da sottolineare è che $\mathrm{i}$ profili molecolari dei ceppi ambientali nosocomiali non correlano con quelli clinici FC; tale dato ci permette di escludere anche il nosocomio come fonte di trasmissione, almeno nei centri FC presenti in questo studio. Viceversa abbiamo dimostrato un alto grado di biodiversità tra ceppi analizzati (indice Simpson range 0.02-0.18) confermando quindi che nel nostro paese, non vi è una presenza di cloni prevalenti nell'ambiente o di cloni che preferibilmente colonizzano i pazienti FC. L'unico caso di correlazione genetica ritrovato riguarda $\mathrm{i}$ ceppi isolati da 5 pazienti, due afferenti al Centro FC di Ancona e 3 al Centro FC di Soverato, in cui è stato trovato un ceppo clonale (GCI-FC). È stato indagato se il ceppo fosse stato acquisito mediante trasmissione interpersonale, ma l'analisi delle cartelle cliniche consultate non ha portato a dimostrare che siano avvenuti contatti tra pazienti all'interno dei centri. Questo genotipo inoltre non appartiene a nessuna linea clonale diffusa nell'ambiente naturale o nosocomiale italiano e/o europeo: infatti, l'analisi mediante MLST dimostra che la sequenza tipo risultante non correla con nessun altro clone presente nel database dedicato.

In conclusione possiamo affermare che risultati di questo studio multicentrico escludono la presenza in Italia di $P$. aeruginosa epidemici in grado di diffondersi nella popolazione FC. In tutti i Centri FC arruolati sono stati trovati gruppi di cluster di modeste dimensioni (per la maggior parte di 2 pazienti) che condividono lo stesso genotipo, ciò può indicare un passaggio interpersonale limitato probabilmente avvenuto all'interno dei centri di cura. Questo dato suggerisce la necessità di incrementare l'osservanza delle misure di prevenzione e controllo dell'infezione in tutti i Centri dedicati alla FC. Non è stato possibile dimostrare un'acquisizione ambientale non avendo trovato genotipi comuni tra ceppi ambientali e ceppi FC, ma la presenza di $P$. aeruginosa nell'ambiente nosocomiale e sopratutto nell'ambiente naturale, come le piscine, suggerisce di consigliare comunque ai pazienti affetti da FC delle linee comportamentali di particolare attenzione nella frequentazione di tali luoghi.

\section{BIBLIOGRAFIA}

1. Agodi A, Sciacca A, Campanile F, et al. Molecular epidemiology of Pseudomonas aeruginosa from cystic fibrosis in Sicily: genome macrorestriction analysis and rapid PCR-ribotyping. New Microbiol. 2000; Jul 23(3): 319-27.

2. Campana S, Taccetti G, Ravenni N, et al. Molecular epidemiology of Pseudomonas aeruginosa, Burkholderia cepacia complex and methicillin-resistant Staphylococcus aureus in cystic fibrosis center. $J$ Cyst Fibros. 2004; 3 (3): 159-63.

3. Campana S, Taccetti G, Ravenni N, et al.Transmission of Burkholderia cepacia complex: evidence for new epidemic clones infecting cystic fibrosis patients in Italy. J Clin Microbiol 2005; 43 (10): 5136-42.

4. Curran B, Jonas D, Grundmann H, Pitt T, Dowson CG. Development of a multilocus sequence typing scheme for the opportunistic pathogen Pseudomonas aeruginosa. J Clin Microbiol. 2004; 42: 5644-9.

5. Dinesh SD, Grundmann H, Pitt TL, Römling U. European wide distribution of Pseudomonas aeruginosa clone C. Clin Microbiol Infect 2003; 9: 1228-33.

6. Doléans-Jordheim A, Cournoyer B, Bergeron E, et al. Reliability of Pseudomonas aeruginosa semi-automated rep-PCR genotyping in various epidemiological situations. Eur J Clin Microbiol Infect Dis. 2009; May 16.

7. Gibson RL, Burns JL, Ramsey BW. Pathophysiology and management of pulmonary infections in cystic fibrosis. Am J Respir Crit Care Med 2003; 5;168 (8): 918-51.

8. Giordano A, Magni A, Graziani C, Sessa R, Quattrucci S, Cipriani P. AP PCR typing of Pseudomonas aeruginosa isolated from patients with cystic fibrosis. New microbiol 2001; 24: 157-63.

9. Griffiths AL, Jamsen K, Carlin JB, et al. Effects of segregation on an epidemic strain in a cystic fibrosis clinic. Am J Resp Crit Care Med 2005; 171: 1020-5.

10. Gruppo di lavoro della Società italiana per lo Studio della Fibrosi Cistica (Festini F. et al.). Raccomandazioni per il controllo e la prevenzione delle infezioni da patogeni respiratori in Fibrosi Cistica. 2006 Ed. SIFC.

11. Gruppo Professionale dei Microbiologi della SIFC (Campana S, Cariani L, Favari F, Garlaschi ML, Fiscarelli E, Manno G). Raccomandazioni per la corretta esecuzione delle indagini microbiologiche in pazienti con Fibrosi Cistica. Ed. Società Italiana Fibrosi Cistica. 2009.

12. Hafiane A, Ravaoarinoro M. Various typing methods of Pseudomonas aeruginosa strains isolated from cystic fibrosis patients. Med Mal Infect. 2008; 38 (5): 238-47.

13. Johnson JK, Arduino SM, Stine OC, Johnson JA, Harris AD. Multilocus sequence typing compared to pulsed-field gel electrophoresis for molecular typing of Pseudomonas aeruginosa. J Clin Microbiol. 2007; 45 (11): 3707-12.

14. Jones AM, Govan JRW, Doherty CJ, Dodd ME, Isalska BJ, Stanbridge TN, Webb AK.. Identification of airborne dissemination of epidemic multiresistant strains of Pseudomonas aeruginosa at a CF centre during a cross infection outbreak. Rev Thorax 2003; 58: 525-7.

15. Leone I, Chirillo MG, Raso T, Zucca M, Savoia D. Phenotypic and genotipi characterization of Pseudomonas aeruginosa from cystic fibrosis patients. Eur J Clin Microbiol Infect Dis 2008; 27: 1093-9.

16. Manno G, Dalmastri C, Tabacchioni S, et al. Epidemiology and clinical course of Burkholderia cepacia complex infections, particularly those caused by different Burkholderia cenocepacia strains, among patients attending an Italian Cystic Fibrosis Center. $J$ Clin Microbiol. 2004; 42 (4): 1491-7.

17. McCallum SJ, Corkill JE, Gallagher MJ. Superinfection with a trasmissibile stranin of Pseudomonas aeruginosa strain in adults with cystic 
fibrosis chronically colonised by $P$. aeruginosa. Lancet. 2001; 358: 558-60.

18. Mena KD, Gerba CP. Risk assessment of Pseudomonas aeruginosa in water. Rev Environ Contam Toxicol 2009; 201: 71-115.

19. Murray TS, Egan M, Kazmierczak BI. Pseudomonas aeruginosa chronic colonization in cystic fibrosis patients. Curr Opin Pediatr 2007; 19 (1): 83-8.

20. Muscarella LF. Contribution of tap water and environmental surfaces to nosocomial transmission of antibiotic-resistant Pseudomonas aeruginosa. Infect Control Hosp Epidemiol 2004; 25 (4): 342-5.

21. O'Carroll MR, Syrmis MW, Wainwright CE, et al. Clonal strains of Pseudomonas aeruginosa in paediatric and adult cystic fibrosis units. Eur Respir J. 2004; 24(1): 101-6.

22. O'Sullivan BP, Freedman SD. Cystic fibrosis. Lancet. 2009; 30-373 (9678): 1891-904.

23. Rademaker JLW, de Bruijn FJ. Characterization and classification of microbes by REP-PCR genomic fingerprinting and computer assisted pattern analysis. In Cactano-Anollès G. and Gresshoff P. M. (ed.), DNA markers: protocols, applications and overviews. $J$ Wiley and Sons, New York. 1997; 151-71.

24. Rajan S, Saiman L. Pulmonary infections in patients with cystic fibrosis. Semin Respir Infect 2002; 17 (1): 47-56.

25. Römling U, Schmidt KD, Tümmler B. Large genome rearrangements discovered by the detailed analysis of 21 Pseudomonas aeruginosa clone $\mathrm{C}$ isolates found in environment and disease habitats. J Mol Biol. Aug
1997; 22; 271(3): 386-404.

26. Scott FW, Pitt TL. Identification and characterization of transmissible Pseudomonas aeruginosa strains in cystic fibrosis patients in England and Wales. $J$ Med Microbiol. 2004; 53 (Pt 7): 609-15.

27. Speert DP, Campbell ME, Henry DA. Epidemiology of Pseudomonas aeruginosa in cystic fibrosis in British Columbia, Canada. Am J Respir Crit Care Med. 2002; 1: 988-93.

28. Speert DP. Front Biosci. Molecular epidemiology of Pseudomonas aeruginosa 2002; 1;7: e354-61.

29. Starkey M, Hickman JH, Ma L, et al. Pseudomonas aeruginosa rugose small-colony variants have adaptations that likely promote persistence in the cystic fibrosis lung. J Bacteriol 2009; 27;191 (11): 3492503.

30. Stover CK, Pham XQ, Erwin AL, et al. Complete genome sequence of Pseudomonas aeruginosa PAO1, an opportunistic pathogen. Nature. Aug 2000; 31; 406(6799): 959-64.

31. Syrmis MW, O'Carroll MR, Sloots TP, et al. Rapid genotyping of Pseudomonas aeruginosa isolates harboured by adult and paediatric patients with cystic fibrosis using repetitive-elements-based PCR assays. J Med Microb 2004; 53: 1089-96.

\section{Acknowledgements}

This study was supported by the "Italian Cystic Fibrosis Foudation - Verona" with the grants FFC\#8/2005 e FFC\#12/2007 "Business group Bologna \& Ferrara", "FFC Delegation Ferrara" and "In Job" Society. 